\title{
VALIDAÇÃO DE TÉCNICA HEMOSTÁTICA DO COMPLEXO ARTERIOVENOSO OVARIANO NA OVARIOSALPINGOHISTERECTOMIA DE GATAS
}

\section{HEMOSTASIS TECHNIQUE VALIDATION OF OVARIAN ARTERIAL VENOUS COMPLEX IN QUEENS OVARIOSALPINGOHISTERECTOMY}

\author{
Camila Pinho Balthazar da Silveira ${ }^{1}$ \\ Marília Silva Carneiro Araújo² \\ Mônica Horr ${ }^{1}$ \\ Elane Arrais Machado Alencar ${ }^{3}$ \\ Roberta Rigaud ${ }^{1}$ \\ Arianne Pontes Oriá ${ }^{4}$ \\ Emanoel Ferreira Martins Filho ${ }^{1}$ \\ João Moreira Costa Neto ${ }^{4}$ \\ 1Pós Graduandos da Universidade Estadual Paulista, Jaboticabal, SP, Brasil. \\ 2Pós Graduanda da Universidade Federal da Bahia, Salvador, BA, Brasil. \\ ${ }^{3}$ Pós Graduanda da União Metropolitana de Educação e Cultura, Lauro de Freitas, BA, Brasil. \\ ${ }^{4}$ Professores Doutores da Universidade Federal da Bahia, Salvador, BA, Brasil - profjmcn@gmail.com
}

\section{Resumo:}

O estudo objetivou descrever a técnica de obliteração do complexo arteriovenoso ovariano (CAVO) por meio da confecção de um nó sob seu próprio eixo, além de avaliar a eficácia desta técnica hemostática na ovariosalpingohisterectomia de gatas, bem como averiguar os tempos parciais de obliteração dos CAVOs e o tempo total do procedimento. Após treinamento prévio, com auxílio de métodos alternativos para ganho de habilidade cirúrgica, foram realizados procedimentos cirúrgicos in vivo. Realizou-se estudo experimental com finalidade de comparar a técnica proposta com a técnica de ligadura com três pinças hemostáticas. O treinamento in vivo, inicialmente em gatas hígidas e, posteriormente, em portadoras de afecções uterinas, serviu para consolidar a destreza adquirida por meio de métodos alternativos e para averiguar a exequibilidade da técnica descrita, que se mostrou eficaz. No estudo comparativo não houve diferença estatística significativa entre o tempo cirúrgico $(\mathrm{p}=0,2848)$ e a obliteração do CAVO direito $(\mathrm{p}=0,1036)$ entre os dois grupos, porém foram observadas diferenças significativas no tempo de obliteração do CAVO esquerdo $(\mathrm{p}=0,0001)$. Diante dos resultados obtidos a partir das avaliações realizadas no período de observação transoperatória, podemos inferir que a técnica descrita é exequível e promove adequada hemostasia do CAVO.

Palavras-chave: cirurgia; felino; obliteração vascular.

\begin{abstract}
:
The present study aimed at describing ovarian arterial venous complex (OVAC) obliteration technique consisting in tying a knot over its own axis. It also aimed at evaluating the efficacy of this hemostatic technique in queens ovariosalpingohisterectomy, as well as checking partial OVAC obliteration time and total procedure time. After previous training - based on alternative methods for the demanded surgical skills acquisition, in vivo surgical procedures were conducted. Once gathered the necessary skills, an experimental study was carried in order to compare this technique
\end{abstract}


with the linking technique in which three hemostatic clamps are used. In vivo training, firstly applied in healthy queens, and later in uterine affection carriers, helped consolidating the dexterity previously acquired through alternative practice methods, and also contributed to the feasibility verification of the described technique, which showed to be effective. Comparative study did not reveal any significant statistic difference between both groups on total surgical elapsed time ( $p$ $=0.2848)$ and time spent for the right OVAC obliteration $(\mathrm{p}=0.1036)$, however significant difference was observed on the left OVAC obliteration $(\mathrm{p}=0.0001)$. The results obtained during transoperatory observation led to the conclusion that the described technique is feasible and provides appropriate OVAC hemostasis.

Keywords: feline; surgery; vascular obliteration.

Recebido em:06 ago. 2012.

Aceito em 18 nov. 2014.

\section{Introdução}

A ovariosalpingohisterectomia (OSH) é considerada um procedimento cirúrgico de rotina utilizado na Medicina Veterinária, que consiste na realização de uma celiotomia com ablação dos ovários, trompas e útero $^{(1)}$. A OSH em programas de controle populacional de cadelas e gatas aparece como uma das técnicas cirúrgicas de escolha para a contracepção ${ }^{(1-3)}$, devendo, nestes casos, mostrar-se rápida, efetiva, segura e de baixo custo. A OSH também tem sua importância nos partos distócicos ${ }^{(1,4)}$, na prevenção de neoplasias mamárias ${ }^{(5)}$ e na prevenção e no tratamento das doenças do trato reprodutivo ${ }^{(6-9)}$.

$\mathrm{O}$ acesso abdominal retroumbilical pela linha mediana ventral é a abordagem tradicionalmente utilizada, contudo são descritas variações nas manobras cirúrgicas relacionadas ao acesso abdominal, exposição dos órgãos e obliteração vascular, todas, em sua maioria, visando reduzir o tempo cirúrgico e minimizar o trauma tecidual ${ }^{(1,10,11)}$. As vias de acesso mais utilizadas para a realização da ovariosalpingohisterectomia são as da linha média ventral ${ }^{(1)}$, lateral do abdômen (flanco $^{(10)}$ e através de portais destinados à videolaparoscopia ${ }^{(11)}$, neste último caso demandando maior custo.

Em animais de companhia, diversos métodos hemostáticos têm sido empregados para obliteração da vasculatura dos órgãos envolvidos na realização da $\mathrm{OSH}$; dentre eles, ressalta-se a confecção de ligaduras e transfixações que são realizadas com diversos materiais, tais como fios cirúrgicos absorvíveis e não absorvíveis ${ }^{(12)}$, ou artesanais do tipo náilon de pesca ${ }^{(13,14)}$, artefatos como braçadeira de náilon ${ }^{(14-16)}$ e clipes metálicos ${ }^{(11,17)}$.

Um vídeo divulgado na rede mundial de computadores e intitulado "Cat spay in five minutes" demonstra a execução de uma OSH em gata com o emprego de uma manobra que possibilita a hemostasia do complexo arteriovenoso ovariano (CAVO) sem o uso de fios de sutura ou outro material $^{(18)}$. O complexo de vasos é obliterado por meio de uma manobra que realiza a confecção de um nó sobre seu próprio eixo ${ }^{(18)}$. Descrição similar foi feita para obliteração do plexo pampiniforme e ducto deferente na orquiectomia em felinos ${ }^{(12)}$, porém não há, na literatura consultada, referência sobre este tipo de obliteração vascular em cadelas ou gatas.

Considerando a exequibilidade da manobra e a inexistência de referências literárias sobre a mesma, buscou-se descrever a técnica e validar sua eficácia na ovariosalpingohisterectomia de gatas, comparando-a com a técnica tradicional (técnica das três pinças hemostáticas e ligadura para hemostasia do CAVO). 


\section{Material e Métodos}

A pesquisa foi conduzida em duas fases. Na primeira, o vídeo foi analisado e métodos alternativos foram desenvolvidos para adequação da metodologia e aquisição da habilidade cirúrgica necessária para a execução da técnica proposta. Posteriormente, foi realizado treinamento in vivo com 24 gatas, sendo 12 hígidas (grupo hígido) e 12 portadoras de alterações reprodutivas em útero e ovário (grupo doente). A segunda fase, na qual foram utilizadas 22 gatas hígidas, compreendeu um estudo comparativo entre a técnica tradicional (Grupo A) e a técnica proposta (Grupo B).

O experimento foi aprovado pelo Comitê de Ética e Uso de Animais da Escola de Medicina Veterinária e Zootecnia - UFBA, sob o registro 09/2010.

Inicialmente, na primeira fase, para compreensão das manobras necessárias à confecção do nó, foi empregado um recurso composto por um cordão de poliéster trançado, fixado em uma base. Com uma das mãos, manipulava-se o cordão e com a outra, uma pinça hemostática, executando-se manobras pertinentes à rotação do cordão sobre seu próprio eixo $\left(360^{\circ}\right)$ e fixação da pinça transversalmente ao seu seguimento proximal. Em seguida, foi empregado outro recurso, confeccionado a partir de uma garrafa plástica transparente (de polietileno tereftalato) de um litro e duas bexigas tipo canudo de látex, que mimetizavam, respeitando-se a anatomia topográfica, a cavidade abdominal, os ovários e o útero de uma gata (Figura 01 - A).

A manobra foi realizada no segundo recurso. Mimetizando uma ovariosalpingohisterectomia em gata. Para confecção do nó, o ovário e respectivo corno uterino foram tracionados por meio de uma incisão mediana ventral pré-umbilical e o pedículo vascular individualizado (Figura 01 - B). Com o auxílio de uma pinça hemostática, em angulação de $45^{\circ}$, realizava-se a rotação do pedículo sobre seu próprio eixo $\left(360^{\circ}\right)$ e a fixação da ponta da pinça transversalmente ao seguimento proximal do pedículo. Aplicava-se uma segunda pinça transversalmente ao pedículo, em localização próxima ao ovário e o pedículo era seccionado acima da pinça hemostática e o nó confeccionado a partir da tração da alça formada pela rotação do pedículo em seu próprio eixo, em direção à extremidade da pinça (Figura 01 - C, D e E).

Fizeram parte do treinamento graduandos do último ano e médicos veterinários residentes. $\mathrm{O}$ treinamento transcorreu ao longo de uma semana, com exercícios de 01 hora por dia.

Garantidas as habilidades necessárias para a realização da técnica hemostática, foram selecionadas 24 gatas para o estudo, sendo 12 hígidas (grupo hígido) e 12 portadoras de alterações reprodutivas em útero e ovário (grupo doente), de diferentes raças, com idades entre sete meses e cinco anos e peso entre 02 e $05 \mathrm{~kg}$, encaminhadas ao Setor de Cirurgia de um Hospital Veterinário Escola, para contracepção cirúrgica. Os proprietários assinaram termo de consentimento livre e esclarecido para compor o experimento. 


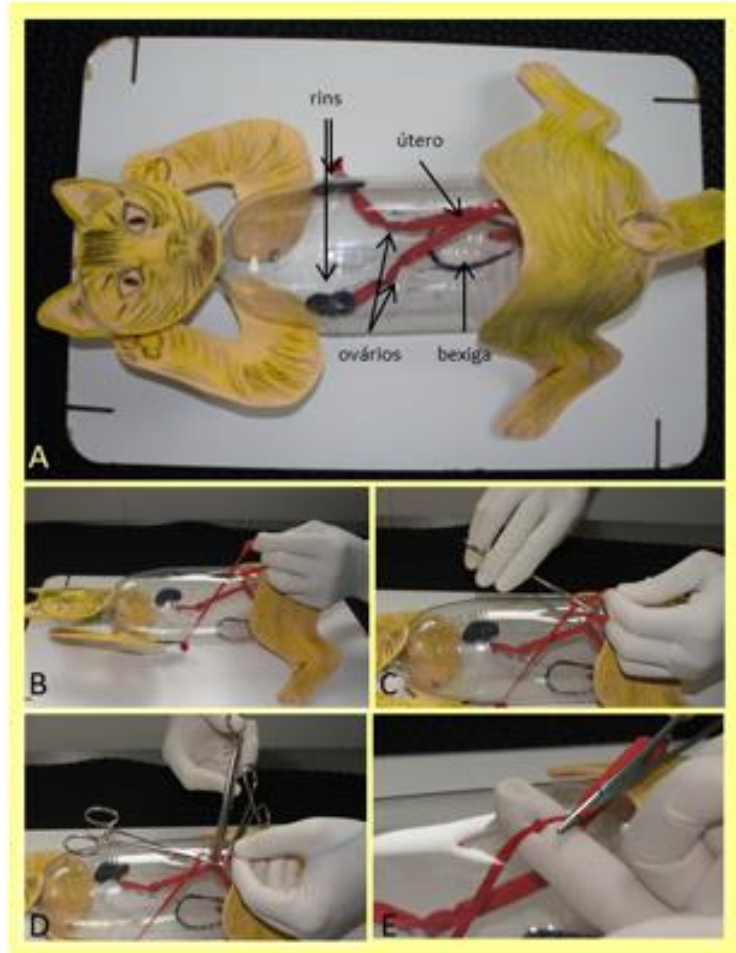

Figura 01: Método alternativo, confeccionado a partir de uma garrafa plástica transparente (de polietileno tereftalato) de um litro e bexigas tipo canudo de látex. Em $\mathrm{A}$, detalhes do sistema genito-urinário mimetizados pelas bexigas de látex. Em B, tração e exposição do ovário direito e respectivo corno uterino, com distensão do pedículo vascular. Com o auxílio de uma pinça hemostática, em angulação de $45^{\circ}$, realizava-se a rotação do pedículo sobre seu próprio eixo e fixação da ponta da pinça transversalmente ao seguimento proximal do pedículo, aplicação de uma segunda pinça transversalmente ao pedículo, em localização próxima ao ovário secção do pedículo e confecção do nó (C, D e E).

O pré-operatório constituiu-se de jejum alimentar de 12 horas e hídrico de seis horas, sendo conduzida terapia antimicrobiana à base de amoxicilina triidratada $(15 \mathrm{mg} / \mathrm{kg}$, por via intramuscular - Agemox, Agener, São Paulo, SP, Brasil) e aplicação de meloxicam (0,1 mg/kg, por via subcutânea - Maxicam, Ouro fino, São Paulo, SP, Brasil). Como protocolo pré-anestésico (MPA), foi utilizada solução de acepromazina a 0,1\% (Acepromazina 0,1\%, São Paulo, SP, Brasil) e cloridrato de tramadol (Cloridrato de tramadol $(50 \mathrm{mg} / \mathrm{mL})$, São Paulo, SP, Brasil ), nas dosagens respectivas de $0,05 \mathrm{mg} / \mathrm{kg}$ e $3 \mathrm{mg} / \mathrm{kg}$, associadas em uma seringa e administradas por via intramuscular. A indução anestésica se deu por propofol (Propofol, SP, Brasil), na dosagem de 4mg/kg, por via intravenosa, e a manutenção da anestesia foi realizada com isofluorano a 2,5\% vaporizado em oxigênio, em circuito aberto com reinalação de gases.

Cabe ressaltar que, para o grupo doente, a escolha da MPA e da terapia pré e transoperatórias estava condicionada ao quadro geral de cada paciente. Caso ocorresse algum tipo de complicação durante a realização da técnica, seria obrigatório abortá-la e proceder à melhor manobra cirúrgica para contornar a situação.

Os animais foram posicionados em decúbito dorsal e a região abdominal ventral foi preparada para a cirurgia asséptica. Em seguida, foram submetidos à celiotomia por meio de incisão mediana 
ventral, retro-umbilical, iniciando-a a um centímetro caudal à cicatriz umbilical, com extensão não superior a três centímetros.

O corpo do útero foi identificado com auxílio do dedo indicador, guiado pela sua localização dorsal à bexiga e ventral ao colón. Ao se seguir a direção cranial, os cornos uterinos direito e esquerdo foram identificados. Com o corno uterino direito exteriorizado, identificou-se o ligamento suspensório, a bursa ovariana e o CAVO (Figura 02 - A). O ligamento suspensório e o ligamento largo foram rompidos (Figura 02 - A e B), formando-se um pedículo vascular composto pelo CAVO. Elevou-se, com uma das mãos, o corno uterino direito e sua respectiva bursa ovárica, sem exercer tração excessiva, estendendo-se o pedículo vascular (Figura 2 - C e D). Com o auxílio de uma pinça hemostática Halstead curva, em angulação de $45^{\circ}$, realizou-se a rotação do pedículo sobre seu próprio eixo $\left(360^{\circ}\right)$ e a fixação da ponta da pinça transversalmente ao seguimento proximal do pedículo (Figura 02 - E e F). Em seguida, uma segunda pinça hemostática foi aplicada transversalmente ao pedículo, em localização próxima ao ovário (Figura 03 - A). O pedículo foi seccionado acima da pinça hemostática de Halstead e o nó confeccionado a partir da tração da alça formada pela rotação do pedículo em seu próprio eixo, em direção à extremidade da pinça, com o auxílio ou não de uma gaze (Figura 03 - B e C). A presença ou não de hemorragia foi avaliada e a hemostasia confirmada.

O corno uterino direito foi retrofletido e o ligamento largo correspondente rompido. A partir do seu deslocamento, identificou-se o corno contralateral e as manobras foram novamente executadas, para oclusão do pedículo ovariano esquerdo (Figura 03 - D e E). Com o corpo do útero exposto, em todos os animais, foi realizada a ligadura para obliteração das artérias e veias uterina direita e esquerda, respectivamente, seguida de transfixação do corpo do útero, com fio categute cromado $\mathrm{n}^{\circ}$ 2-0, cranialmente a cérvix (Figura $03-$ F). Uma pinça hemostática foi colocada transversalmente ao corpo do útero e este foi seccionado, sendo avaliada a presença ou não de hemorragia e, após a confirmação da hemostasia, realizada a omentopexia sobre o coto uterino. Procedeu-se à celiorrafia, com fio mononáilon $\mathrm{n}^{\circ}$ 2-0 com pontos em " $\mathrm{X}$ " para musculatura e ponto simples separados para a redução do espaço morto e síntese da pele. Os animais foram mantidos em observação até normalização dos parâmetros fisiológicos e, em seguida, receberam alta hospitalar. No pósoperatório, prescreveu-se: amoxicilina triidratada, por via intramuscular, a cada 48 horas, totalizando quatro aplicações; meloxicam na dose de $0,1 \mathrm{mg} / \mathrm{kg}$ a cada 24 horas, por via oral, durante 3 dias; e dipirona $25 \mathrm{mg} / \mathrm{kg}$ a cada 8 horas, por 3 dias. Os pacientes foram liberados seis horas após a realização do procedimento cirúrgico, sem resquício de qualquer complicação.

Avaliações clínicas, de ambos os grupos, foram feitas aos dois, sete e dez dias após a cirurgia, com o intuito de avaliar estado geral do paciente e complicações cicatriciais. 


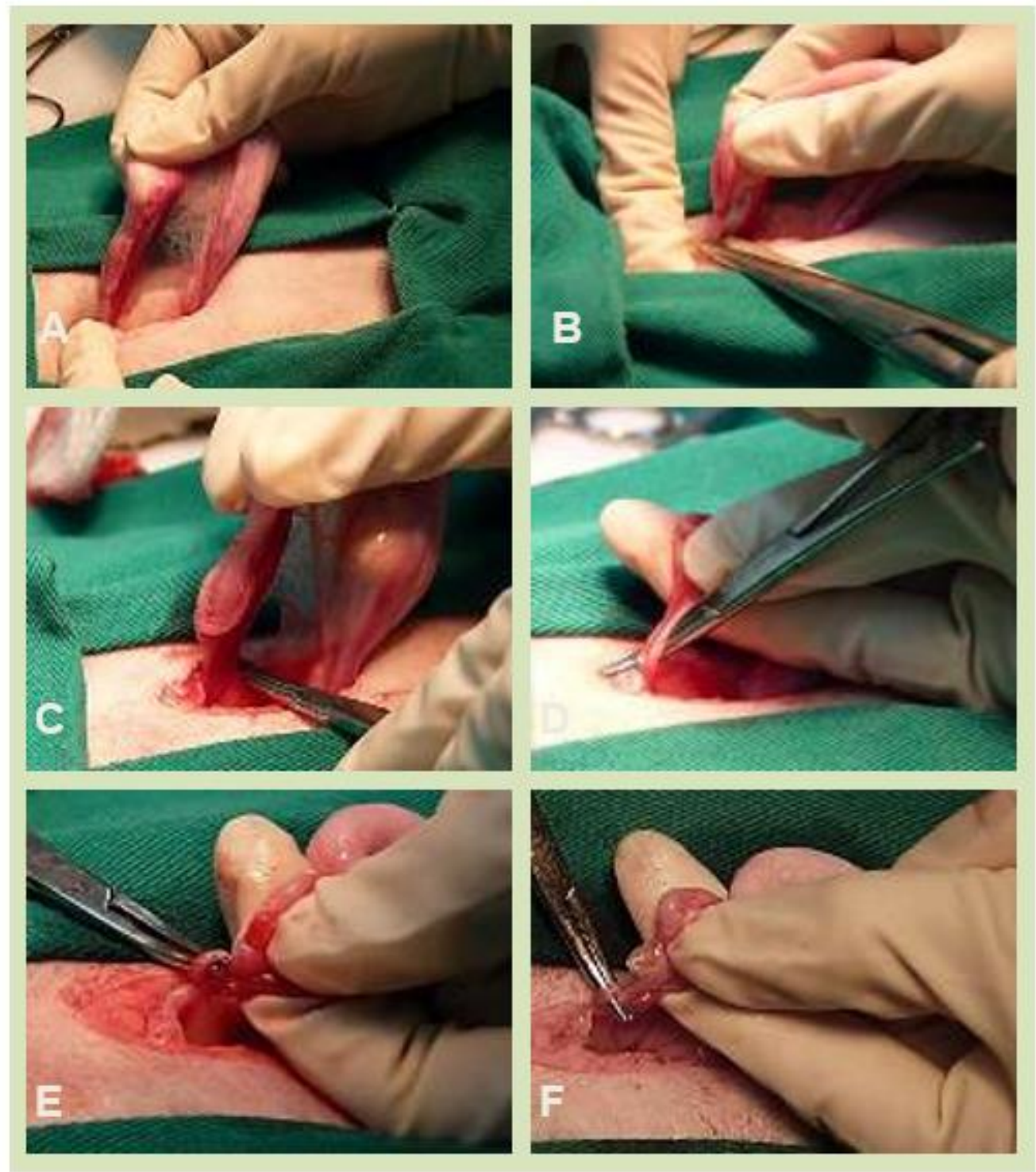

Figura 02: Técnica de hemostasia do complexo arteriovenoso ovariano para ovariosalpingohisterectomia de gatas. Imagens referentes a obliteração do complexo arteriovenoso ovariano por meio da confecção de um nó sobre seu próprio eixo. Em (A), exposição do corno uterino direito e respectiva bursa ovárica e ligamentos; em (B) ruptura do ligamento suspensor empregando-se secção com tesoura; Em (C), ruptura do ligamento largo do útero e formação do pedículo vascular do CAVO; Em (D), (E) e (F), a aplicação da pinça hemostática Halstead curva, em angulação de aproximadamente $45^{\circ}$, para rotação do pedículo sobre seu próprio eixo $\left(360^{\circ}\right)$ e fixação da ponta da pinça transversalmente ao seguimento proximal do pedículo. 


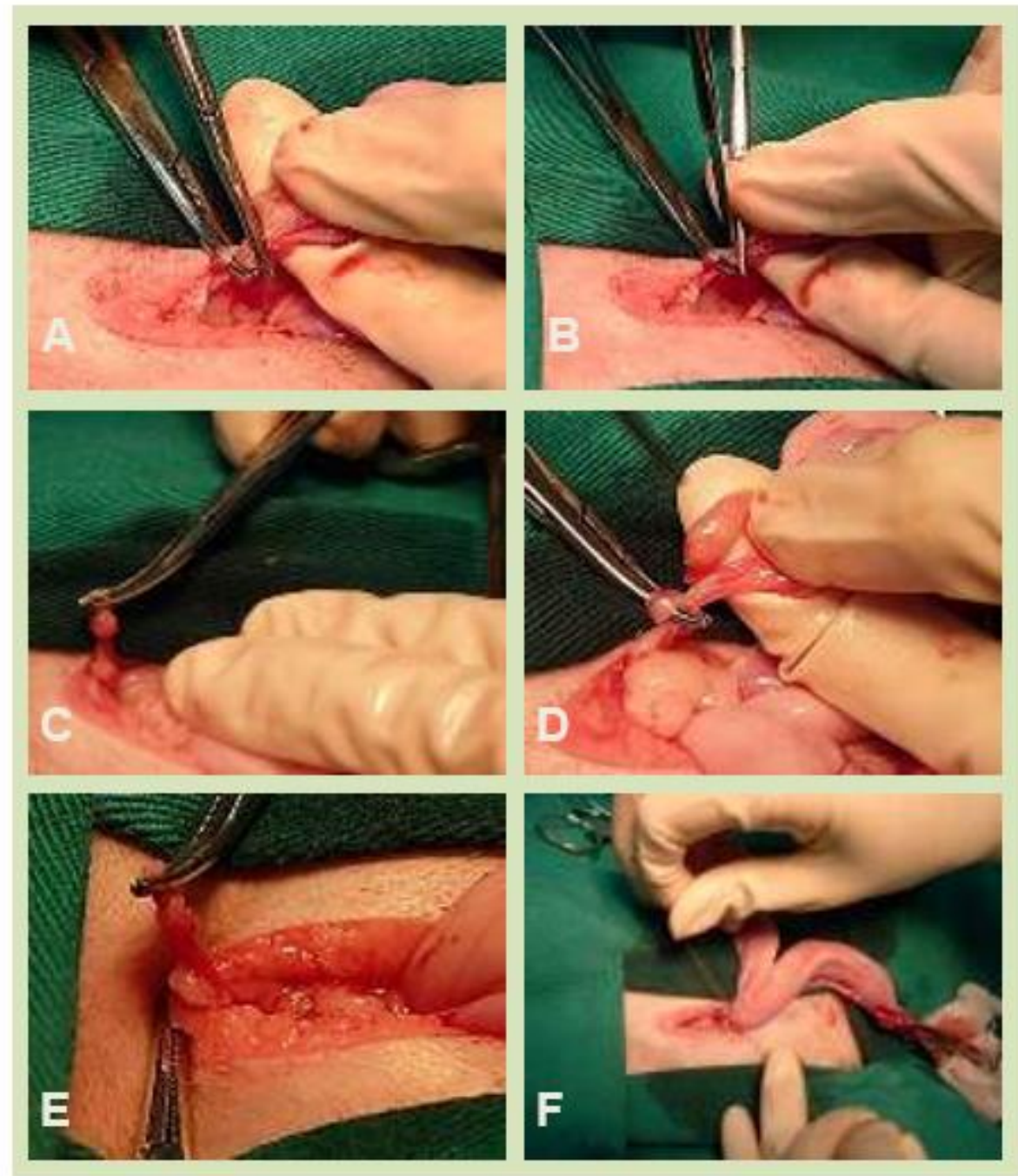

Figura 03: Técnica de hemostasia do complexo arteriovenoso ovariano para ovariosalpingohisterectomia de gatas. Sequência das manobras para obliteração do CAVO por meio da confecção de um nó sobre seu próprio eixo. Em (A), aplicação de uma segunda pinça hemostática colocada transversalmente ao pedículo, em localização próxima ao ovário. $\mathrm{Em}(\mathrm{B}) \mathrm{e}(\mathrm{C})$, secção do pedículo realizada rente à pinça hemostática e conformação do nó após confecção. Em (D) e (E), manobras para confecção do nó no pedículo vascular esquerdo. Em $(F)$, confecção da transfixação do corpo do útero, empregando-se o fio categute $n^{\circ} 2-0$.

$\mathrm{Na}$ segunda fase foi realizado estudo comparativo, em gatas, com o intuito de comparar a técnica descrita com a tradicional, que utiliza três pinças hemostáticas e ligadura para hemostasia do CAVO. Foram selecionados 22 animais hígidos, distribuídos aleatoriamente em dois grupos experimentais de igual número. O Grupo A - composto por onze gatas submetidas a OSH pela técnica tradicional - e o Grupo B - composto por onze gatas submetidas a OSH pela técnica proposta.

Todos os animais foram selecionados pelos mesmos critérios e submetidos a procedimentos perioperatórios idênticos, anteriormente descritos para o treinamento in vivo. Em todos os procedimentos in vivo a equipe cirúrgica foi a mesma. 
Os tempos totais do procedimento e de obliteração do CAVO direito e esquerdo foram registrados para os dois grupos. O tempo total iniciava-se no momento da incisão da pele e se estendia até o fechamento do último ponto aplicado à pele. O tempo de obliteração do CAVO direito e esquerdo tinha início no momento de sua exposição, estendendo-se até a completa confecção do nó e sua liberação na cavidade abdominal.

A análise dos dados foi consignada de forma descritiva para o grupo do treinamento in vivo. Já para o estudo comparativo, realizou-se o teste " $\mathrm{T}$ " de student - comparando-se os tempos envolvidos durante a intervenção cirúrgica com o objetivo de comparar os tempos médios entre os grupos em cada procedimento. Os dados foram apresentados como média \pm erro padrão da média. O nível de significância estabelecido foi de 5\%.

\section{Resultados e Discussão}

Após análise, em vídeo, da técnica cirúrgica para realização de uma $\mathrm{OSH}$, em gata $^{(18)}$, e verificação das manobras para obliteração do CAVO, constatou-se que o método foi exequível e eficaz. Devido à ausência de referências literárias sobre sua descrição ou uso, buscou-se, inicialmente, o treinamento das referidas manobras em recursos substitutivos.

Há uma tendência de buscar métodos alternativos para o aprendizado de técnicas cirúrgicas. A utilização de cadáveres, simuladores e recursos alternativos para a aquisição de habilidade e destreza tem se tornado cada vez mais comum na área médica, particularmente para o ensino de cirurgia $^{(19,20)}$. Neste estudo, os recursos alternativos permitiram o desenvolvimento de habilidades e a destreza necessária para a realização das manobras ora propostas. $\mathrm{O}$ treinamento repetitivo e a ausência de estresse permitiram a adequada consolidação de competências psicomotoras essenciais para a realização da técnica in vivo. Tais constatações tipificaram a importância dos métodos alternativos na aquisição de habilidades manuais e psicomotoras, como reportado por Griffon et al. $^{(19)}$ e Matera ${ }^{(21)}$.

A destreza adquirida foi fundamental para a realização das manobras transoperatórias no treinamento in vivo. Não houve dificuldades para divulsionamento ou exposição do pedículo vascular formado pelo CAVO, devidamente individualizado do ligamento suspensor e do mesovário. Porém, a fragilidade desses pedículos tornou necessária a realização de manobras delicadas e precisas para a confecção do nó proposto, o que somente foi possível mediante treinamento prévio.

No decorrer do treinamento in vivo, as nuances entre a destreza adquirida a partir dos recursos alternativos e sua efetiva aplicação nos animais mostraram-se evidentes, necessitando de ajustes e maiores cuidados, como por exemplo, a necessidade de, no momento da rotação da pinça hemostática sobre o pedículo, manter sua inclinação em um ângulo de aproximadamente $45^{\circ}$. Quando não realizada ou feita de maneira incorreta, tal manobra dificultava a confecção do nó, acarretando tentativas que quase sempre fragilizavam o tecido, impossibilitando a aplicação da técnica. Isto ocorreu com quatro pedículos, sendo necessária a conversão para a técnica tradicional. Nesta fase, em 66,6\% dos animais hígidos houve determinado grau de dificuldade quando da execução das manobras. A partir do oitavo animal, pode-se perceber que as complicações tornaramse menos frequentes. À medida que a equipe cirúrgica avançava em número de procedimentos, melhorava o desempenho cirúrgico, observação condizente com o desenvolvimento de competência 
psicomotora e pela curva de aprendizado descrita por outros autores ${ }^{(19,20)}$.

No grupo de animais doentes, apesar das alterações patológicas observadas nos órgãos, contou-se, nesta fase, com uma maior experiência do executor. Além disso, animais com alterações no útero em decorrência de gestação, especialmente no terço final, apresentam maior relaxamento dos ligamentos pélvicos, devido à ação do estrógeno e da relaxina, facilitando a sua exposição durante o ato cirúrgico ${ }^{(7)}$. Contudo, os vasos ovarianos e uterinos encontravam-se mais calibrosos, podendo favorecer a ocorrência de hemorragias ${ }^{(7,22)}$. O aumento da vascularização dos órgãos nesse grupo, foi responsável, em quatro animais pela ocorrência de hemorragias, quando da ruptura do ligamento suspensório $^{(16)}$. Embora seja de fácil controle e resolução, empregando-se hemostasia por compressão, há de se conjecturar que a sua ocorrência prolonga o tempo operatório.

O grau de ingurgitamento e o calibre do vaso foram fatores relevantes durante a confecção do nó; assim como foi visto nos animais do grupo hígido, a principal dificuldade residiu na tração da alça do nó sobre a extremidade da pinça. Com o ingurgitamento do vaso, tal manobra tornou-se mais difícil. A partir do momento em que foi utilizada uma compressa de gaze para fixar a alça do pedículo e tracioná-la em direção à extremidade da pinça, esse problema foi contornado.

Apesar de as mesmas alterações terem sido observadas em ambos os grupos, nos animais hígidos, elas poderiam estar relacionadas ao processo de aquisição da habilidade cirúrgica. Nó frouxo ou mesmo repetidas tentativas de confeccioná-lo foram mais frequentes no grupo hígido do que no grupo doente e em ambos os grupos tais complicações ocorreram durante a obliteração do CAVO direito. Autores reportaram ocorrências de complicações em OSH de cadelas executadas por principiantes $^{(23,24)}$, sinalizando a maior frequência de complicações hemorrágicas no pedículo ovariano direito do que no esquerdo, dado que corrobora com os resultados obtidos no presente estudo. Contudo, a falta de trabalhos científicos acerca de treinamento de técnicas e aquisição de habilidade cirúrgica limitam as discussões sobre o assunto.

No estudo comparativo, não foram encontradas diferenças estatísticas significativas entre a técnica tradicional (Grupo A) e a técnica proposta (Grupo B), relativamente ao tempo de obliteração do CAVO direito $(\mathrm{p}=0,1036)$ (Figura $04-\mathrm{A})$ e o tempo total no procedimento cirúrgico $(\mathrm{p}=0,2848)$ (Figura 04 - C). Entretanto, houve diferença estatística significante com relação ao tempo de obliteração do CAVO esquerdo ( $\mathrm{p}=0,0001$ ) (Figura 04 - B).

Foi perceptível, tanto no grupo A quanto no B, o menor tempo de obliteração do CAVO esquerdo em relação ao direito (Figura 04 - A e B). A sua melhor exposição devido à sua posição anatômica mais caudal ao rim ${ }^{(12)}$ facilitou a confecção da hemostasia do CAVO esquerdo, embora a do Grupo B tenha se mostrado superior com relação ao rápido tempo de sua execução (Figura 04 - B). Tal situação não foi encontrada durante a obliteração do CAVO direito em ambos os grupos.

Apesar de não haver diferença estatística significante entre o Grupo A e o Grupo B, com relação ao tempo total no procedimento cirúrgico $(\mathrm{p}=0$,2848) (Figura $04-\mathrm{C})$, na prática, percebeu-se uma tendência a se executar a técnica descrita $(13,80 \pm 1,19)$ de modo mais rápido que a técnica tradicional $(15,34 \pm 0,72)$, desde que não ocorresse complicação no momento de sua execução. É provável que esse resultado se deva ao ganho de habilidade em executar a técnica, bem como à não utilização de fio de sutura nos pedículos, o que possivelmente otimizou o tempo cirúrgico. 

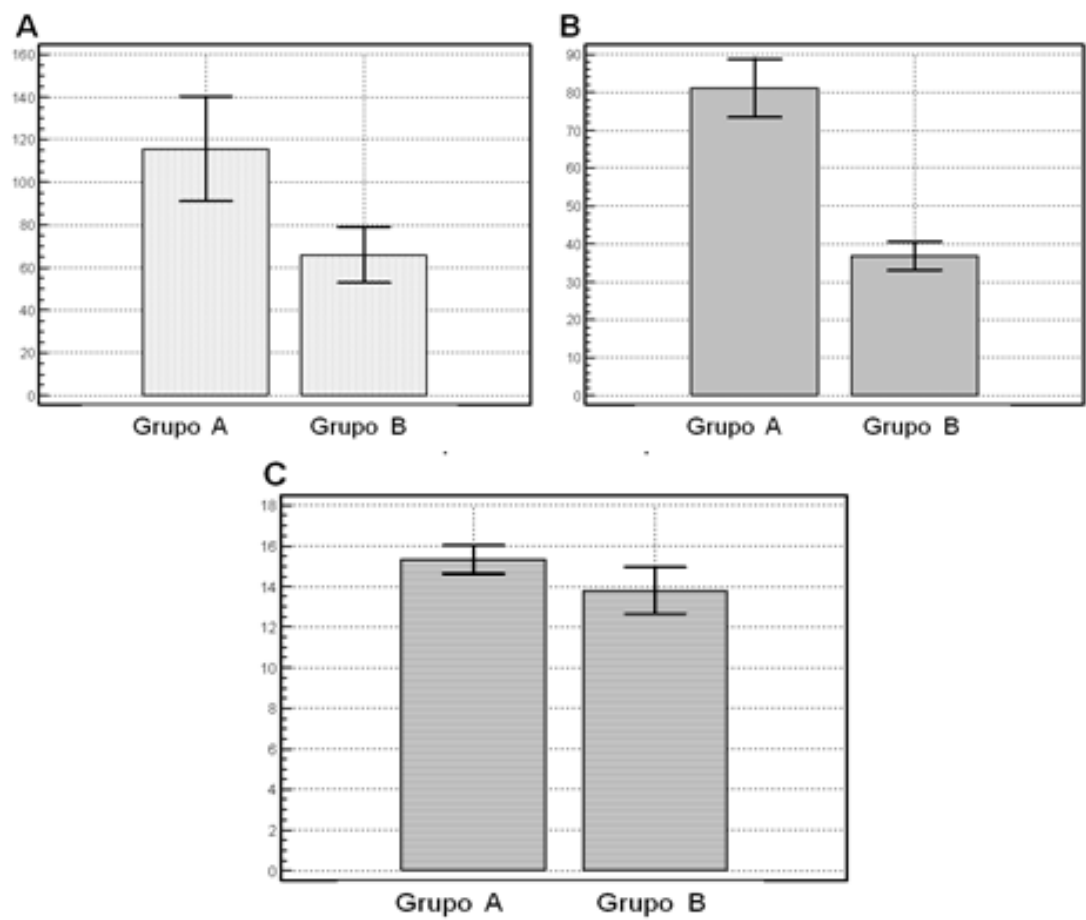

Figura 04: (A) Tempos médios em segundos para obliteração do complexo artériovenoso ovariano direito. (B) Tempos médios em segundos para obliteração do complexo artériovenoso ovariano esquerdo. (C) Tempos médios em minutos para procedimento cirúrgico total. Grupo A, gatas submetidas a ovariosalpingohisterectomia pela técnica tradicional; Grupo B, gatas submetidas a ovariosalpingohisterectomia pela técnica proposta. As caixas representam as médias e as barras os erros-padrão das médias.

Sabe-se que as complicações pós-operatórias relacionadas ao material de sutura empregado no pedículo ovariano e útero podem ocorrer. O fio de sutura pode agir como fator irritativo permanente, podendo exacerbar e cronificar a resposta inflamatória local e causar transtornos à fisiologia da cicatrização, além de predispor à formação de granuloma ${ }^{(25,26)}$. A nova técnica pode ser uma alternativa à técnica tradicionalmente empregada, tanto por diminuir as chances de reação tecidual por presença de corpo estranho, já que não envolve a utilização de material inanimado para a realização da obliteração do CAVO, quanto por demandar menor custo.

No vídeo elaborado por Larson ${ }^{(18)}$, a técnica é realizada com rapidez e destreza. Entretanto, é evidente a falta de preocupação com manobras básicas de hemostasia, como utilização de pinça hemostática. Durante a execução relatada neste trabalho, algumas condutas de hemostasia foram introduzidas com o propósito de tornar o procedimento mais seguro, realizando-se sempre os pinçamentos necessários para conter qualquer tipo de hemorragia. A esse fato pode estar atrelado o maior tempo de execução da técnica no presente trabalho. Outro ponto a considerar é que não foi utilizado o gancho de castração, o que provocou maior abertura na cavidade abdominal e aumento do tempo total da cirurgia. Porém, mesmo sem a utilização do gancho de castração, a extensão da incisão cirúrgica não ultrapassou três centímetros.

Durante toda a execução do experimento não houve histórico de óbito ou complicação no pósoperatório imediato e tardio. 


\section{Conclusão}

Diante dos resultados obtidos das avaliações realizadas no período de observação transoperatória de gatas submetidas à $\mathrm{OSH}$, pode-se inferir que a técnica descrita é exequível e promove adequada hemostasia do CAVO. Quando comparada à técnica hemostática tradicional, mostrou-se segura, rápida e econômica, porquanto dispensa ligaduras ou fios.

\section{Agradecimentos:}

À CAPES e ao Projeto PROCAD NF08 UFBA/UNESP. À Graduanda Larissa Julião e aos Pósgraduandos Marcela Aldrovani, Leandro Ziemer Carneiro e Ana Raquel de Araújo Ferreira.

\section{Referências}

1. Howe LM. Surgical methods of contraception and sterilization. Theriogenology. 2006; 66:500-509. Disponível em http://dx.doi.org/10.1016/j.theriogenology.2006.04.005

2. Van Goethem B, Schaefers-Okkens A, Kirpensteijn J. Making a rational choice between ovariectomy and ovariohysterectomy in the dog: A discussion of the benefits of either technique. Vet Surg. 2006;35(2):136143. Disponível em: http://dx.doi.org/10.1111/j.1532-950X.2006.00124.x

3. Lui JF, Toniollo GH, Savi PAP, Voorwald FA, Silva MAM, Tosta PA. Esterilização cirúrgica de caninos e felinos em Jaboticabal. Interação entre o benefício social e a pesquisa científica. Rev. Ciênc. Ext. 2011;7(2):29-40. Disponível em: http://ojs.unesp.br/index.php/revista_proex/article/view/394/614

4. Luz MR, Freitas PMC, Pereira EZ. Gestação e parto em cadelas: fisiologia, diagnóstico de gestação e tratamento das distocias. Rev. Bras. Reprod. Anim 2005;29(3/4):142-150. Disponível em: http://www.cbra.org.br/pages/publicacoes/rbra/download/pag\%20142\%20v29n3-4.pdf

5. Fonseca CS, Daleck CR. Neoplasias mamárias em cadelas: influência hormonal e efeitos da ováriohisterectomia como terapia adjuvante. Cienc. Rural. 2000;30(4):731-735. Disponível em: http://dx.doi.org/10.1590/S0103-84782000000400030

6. Martins LR, Lopes MD. Pseudociese canina. Rev. Bras. Reprod. Anim. 2005;29(3/4):137-141. Disponível em: http://www.cbra.org.br/pages/publicacoes/rbra/download/pag\%20137\%20v29n3-4.pdf

7. Oliveira ECS, Marques-Jr AP. Endocrinologia reprodutiva e controle da fertilidade da cadela. Rev. Bras. Reprod. Anim. 2006;30(1/2):11-18. Disponível em: http://www.cbra.org.br/pages/publicacoes/rbra/download/RB002\%200liveira\%20p11-18.pdf

8. Pliego CM, Ferreira MLG, Ferreira AMR, Leite JS. Leiomiossarcoma uterino em cadela. Vet. e Zootec. 2008;15 Suppl 3: 40-41. Disponível em: http://www.fmvz.unesp.br/rvz/index.php/rvz/article/download/442/336

9. Santos FC, Corrêa, TP, Rahal SC, Crespilho AM, Lopes MD, Mamprim MJ. Complicações da esterilização cirúrgica de fêmeas caninas e felinas. Revisão da literatura. Vet. e Zootec. 2009;16(1):8-18. Disponível em: www.fmvz.unesp.br/rvz/index.php/rvz/article/download/378/296

10. Minguez RE, Martinez-Darve JG, Cuesta MM. Ovariohisterectomia de gatas e cadelas pelo flanco. Rev. Bras. Reprod. Anim. 2005;29(3/4):151-158. Disponível em: http://www.cbra.org.br/pages/publicacoes/rbra/download/pag\%20151\%20v29n3-4.pdf

11. Schiochet F, Beck CAC, Silva APFF, Contesini EA, Alievi MM, Stedile R, Pinto V, Yamazaki PH, Jurinitz DF, Pellizari M. Ovário-histerectomia laparoscópica em felinos hígidos: estudo comparativo de três 
métodos de hemostasia. Arq. bras. med. vet. zootec. 2009;61(2):369-377. Disponível em: http://dx.doi.org/10.1590/S0102-09352009000200013

12. Migliari R, De Vuono RS. Ovariosalpingohisterectomia em cadelas e gatas: Um novo procedimento é proposto. Rev. Educ. Contin. CRMV-SP. 2000;3(3): 28-32. Disponível em: http://revistas.bvsvet.org.br/recmvz/article/view/3328/2533

13. Rahal SC, Rocha NS, Figueiredo LA. Estudo comparativo das reações teciduais produzidas pela "linha de pesca" (poliamida) e fio de náilon cirúrgico. Cienc. Rural. 1998;28(1):89-93. Disponível em: http://dx.doi.org/10.1590/S0103-84781998000100015

14. Lima AFM, Luna SPL, Rodrigues MMP, Quitzan JG. Avaliação histológica e videolaparoscópica de ligaduras dos pedículos ovarianos realizados com mononáilon agulhado ou abraçadeiras auto-estáticas de náilon em cadelas submetidas à ovariossalpingohisterectomia pela técnica do gancho. Ars vet. 2010;26(2):66-70. Disponível em: http://arsveterinaria.org.br/index.php/ars/article/view/268/226

15. Barros BJ, Sanches AWD, Pachaly JR. Utilização de abraçadeiras de náilon 6.6 (poliamida) como método de ligadura de pedículos ovarianos e coto uterino em ovário-histerectomia eletiva em cadelas (Canis familiaris). Arq. Ciên. Vet. Zool. Unipar 2009;12(1):47-60. Disponível em: http://revistas.unipar.br/veterinaria/article/view/2934

16. Costa Neto JM, Teixeira EM, Ferreira Filho EM, Toríbio JMML, Almeida Filho CHR, Moraes VJ, D'Assis MJMH. Braçadeiras de náilon para hemostasia preventiva na ovariosalpingohisterectomia em gatas. Rev. Bras. Saúde Prod. An. 2009;10(3):615-624. Disponível em: http://revistas.ufba.br/index.php/rbspa/article/view/1450/853

17. Malm C, Savassi-Rocha PR, Gheller VA, Oliveira HP, Lamounier AR, Foltyneck V. Ováriohisterectomia: estudo experimental comparativo entre as abordagens laparoscópica e aberta na espécie canina. Intra-operatório-I. Arquivo Brasileiro de Medicina Veterinária e Zootecnia. 2004;56(4):457-466. Disponível em: http://dx.doi.org/10.1590/S0102-09352004000400006

18. Larson PW. Cat spay in five minutes [arquivo de vídeo]. 2008 ago 26 [Acessado em: 2011 ago 20] Disponível em: http://www.youtube.com/watch?v=LGIiES0jyRs.

19 Griffon DJ, Cronin P, Kirby B, Cottrell DF. Evaluation of a hemostasis model for teaching ovariohysterectomy in Veterinary Surgery. Vet Surg. 2000;29:309-316. Disponível em: http://dx.doi.org/10.1053/jvet.2000.7541

20. Silva RMG, Matera JM, Ribeiro AACM. Preservation of cadavers for surgical technique training. Vet Surg. 2004;33(6):606-608. Disponível em: http://dx.doi.org/10.1111/j.1532-950x.2004.04083.x

21. Matera JM. O ensino de cirurgia: da teoria à prática. Ciênc. vet. tróp, 2008;11 Suppl 1:96-101. Disponível em: http://www.rcvt.org.br/palestras/p93.pdf

22. Smith FO. Canine pyometra. Theriogenology. 2006;66 (3):610-612. Disponível em: http://dx.doi.org/10.1016/j.theriogenology.2006.04.023

23. Burrow R, Batchelor D, Cripps P. Complications observed during and after ovariohysterectomy of 142 bitches at a veterinary teaching hospital. Vet. Rec. 2005;157 (26):829 -833. Disponível em: http://dx.doi.org/10.1136/vr.157.26.829

24. Adin CA. Complications of ovariohysterectomy and orchiectomy in companion animals. Vet Clin Small Anim. 2011;41:1023-1039. Disponível em: http://dx.doi.org/10.1016/j.cvsm.2011.05.004

25. Maldonado F, Muñoz L, Quezada M, Briones M, Urrutia P. Reacción tisular a materiales de sutura no absorbibles en piel de equinos. Arch. med. vet. 2006;38(1):63-67. Disponível em: http://dx.doi.org/10.4067/S0301-732X2006000100009

26. Martins AW, Popak P, Rodrigues CG. Hidronefrose e megaureter em consequência à reação tecidual em cadela pastor alemão - Relato de caso. Vet. not. 2006;12 Suppl 2:95. Disponível em: http://www.seer.ufu.br/index.php/vetnot/article/view/18753 